\title{
NUMERICAL DISCRETIZATION OF COUPLING CONDITIONS BY HIGH-ORDER SCHEMES
}

\author{
MAPUNDI K. BANDA, AXEL-STEFAN HÄCK, AND MICHAEL HERTY
}

\begin{abstract}
We consider numerical schemes for $2 \times 2$ hyperbolic conservation laws on graphs. The hyperbolic equations are given on the spatially one-dimensional arcs and are coupled at a single point, the node, by a nonlinear coupling condition. We develop high-order finite volume discretizations for the coupled problem. The reconstruction of the fluxes at the node is obtained using derivatives of the parameterized algebraic conditions imposed at the nodal points in the network. Numerical results illustrate the expected theoretical behavior.
\end{abstract}

35R02, 35Q35, 35F30

Keywords. numerical methods, higher-order coupling, networks of fluid dynamics,

\section{INTRODUCTION}

We are interested in the high order numerical discretization of flow problems on networks where the dynamics are governed by $2 \times 2$ systems of nonlinear hyperbolic partial differential equations. Among the many examples where such systems arise are traffic flow $[24,25,29,31]$, production networks $[21,23,30]$, telecommunication networks [22], gas flow in pipe networks $[3,11-13,15,16]$ or water flow in canals $[4,5,28,35]$. Mathematically, flow problems on networks are boundary value problems where the boundary value is implicitly defined by a coupling condition. This condition is either physically or mathematically motivated and may consist of algebraic conditions coupling the flow in different arcs or may consist of ordinary differential equations. We are interested in finite-volume methods to resolve the hyperbolic dynamics. Most of the available finite volume methods solve the coupling condition explicitly $[2,8-10]$. For the evolving state at the node, a Godunov-type scheme [2] or kinetic scheme [8] is applied to determine the fluxes at the cell interface. Those schemes are explicit in time and therefore the dynamics on different arcs decouple contrary to an implicit discretization (in time) [33].

We develop a second-order finite volume scheme for general $2 \times 2$ hyperbolic systems on networks. The crucial point is the derivation of a suitable numerical flux at the nodal point where we apply high order reconstruction using temporal derivatives of the state at the node. We use a characteristic decomposition of the temporal derivative of the solution at the nodal point and estimate the outgoing information using spatial derivatives. This procedure has been applied to (pure) boundary value problems in the context of finite differences (and finite volumes) for example in [39-41] and mimics the

Date: December 22, 2015.

University of Pretoria, Pretoria, South Africa mapundi.banda@up.ac.za .

RWTH Aachen University, Aachen, Germany; haeck@igpm.rwth-aachen.de.

RWTH Aachen University, Aachen, Germany; herty@igpm.rwth-aachen.de. 
Cauchy-Kowalewski theorem for sufficiently smooth solutions. It should be mentioned that also in [39] been developed for an arbitrary order of accuracy while we only discuss second-order schemes. We show that the derived scheme coincides with a second-order discretization in the case of two connected arcs, we validate the discretization by reformulating a classical boundary value problem using coupling conditions and we present numerical results for gas flow in pipe networks. The only other approach to high order coupling conditions on networks which we are aware of is [6]. Therein, additional ghost cells for each connected arc are introduced to recover a solution and the information on the temporal derivatives is obtained without characteristic decomposition. We discuss the relation between the schemes in Section 3.

\section{Motivation AND NUMERICAL SCHEME}

As in $[2,3,15,16]$ we consider the following model for flows on graphs. This situation covers examples in gas pipe networks, traffic flow and water flow in open canals. We consider a single vertex connected to $n \operatorname{arcs} j=1, \ldots, n$. The arcs extend to infinity hence they are parameterized by the interval $[0, \infty)$. The vertex is located at $x=0$ for all arcs.

Remark 2.1. The reduction of a network to a single vertex does cover the problem of an arbitrary large graph, since we assume the dynamics on the arcs to be hyperbolic conservation laws. The hyperbolicity conditions provides us with the property of a finite speed of information propagation. Therefore, the solution of the coupling problem at a vertex of the initial network is not coupled with the states at the other vertices.

For simplicity of the presentation, we assume that the flux $f$ is the same on all connected arcs. Let $f \in C^{4}\left(\mathbb{R}^{2} ; \mathbb{R}^{2}\right)$ and $u_{j}(x, t): \mathbb{R}_{0}^{+} \times \mathbb{R}_{0}^{+} \rightarrow \mathbb{R}^{2}$ for $j=1, \ldots, n$ where $u_{j}$ denotes the conserved variables on arc $j$. The dynamics of $u_{j}$ are governed by

$$
\begin{aligned}
\partial_{t} u_{j}+\partial_{x} f\left(u_{j}\right) & =0, t \geq 0, x \geq 0, \\
u_{j}(x, 0) & =u_{j, 0}(x), x \geq 0, \\
\Psi\left(u_{1}(0+, t), \ldots, u_{n}(0+, t)\right) & =0, t \geq 0,
\end{aligned}
$$

where $\Psi: \mathbb{R}^{2 n} \rightarrow \mathbb{R}^{n}$ is the possibly nonlinear coupling condition. The situation is illustrated in Figure 1.

We assume that $\Psi$ fulfills a transversality condition (4) below:

$$
\operatorname{det}\left[D_{1} \Psi(\hat{u}) r_{2}\left(\hat{u}_{1}\right), \ldots, D_{n} \Psi(\hat{u}) r_{2}\left(\hat{u}_{n}\right)\right] \neq 0 .
$$

which ensures well-posedness of problem (1) to (3). Here, $D_{j} \Psi(\hat{u})=\frac{\partial}{\partial u_{j}} \Psi(\hat{u})$ and $\hat{u} \in$ $\mathbb{R}^{2 n}$ is a steady state solution to $(1)$ such that $\Psi(\hat{u})=0$ and $r_{2}$ denotes the eigenvector to the second eigenvalue. We refer to [16, Definition 3.1] and [16, Theorem 3.2] for more details on the definition of the solution and assumption on the characteristic fields as well as the existence and uniqueness result. For convenience we recall the definition of a solution below. Let $\Omega \subset \mathbb{R}^{2}$ non-empty and open set. Let $\bar{u}:=\left(\bar{u}_{1}, \ldots, \overline{u_{n}}\right) \in \Omega^{n}$. Let $f \in C^{4}\left(\Omega ; \mathbb{R}^{2}\right)$ where for each $\ell=1, \ldots, n D f\left(\bar{u}_{\ell}\right)$ admits a strictly negative $\lambda_{1}\left(\bar{u}_{\ell}\right)$ and a strictly positive eigenvalue $\lambda_{2}\left(\bar{u}_{\ell}\right)$, and each characteristic field is either genuinely nonlinear or linearly degenerated. 


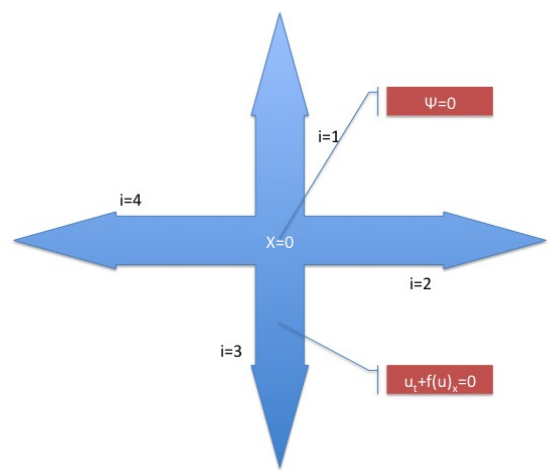

Figure 1. An example of the problem (1) to (3) in the case of $n=4$ connected arcs. For simplicity the arcs are extend to $x=+\infty$. On each arc the dynamics is governed by the nonlinear hyperbolic system (1) as indicated by the legend. The dynamics $u_{i}(0+, t)$ are coupled at a nodal point located at $x=0$ by the nonlinear coupling conditions (3) indicated by the corresponding legend.

Definition 2.2. Fix a map $\Psi \in C^{1}\left(\Omega ; \mathbb{R}^{n}\right)$. A weak solution on $[0, T]$ to

$$
\left\{\begin{array}{lll}
\partial_{t} u_{l}+\partial_{x} f\left(u_{l}\right)=0 & t \in \mathbb{R}^{+} \quad l \in\{1, \ldots, n\} \\
\Psi(u(t, 0))=0 & x \in \mathbb{R}^{+} \quad u_{o} \in \bar{u}+L^{1}\left(\mathbb{R}^{+} ; \Omega^{n}\right) \\
u(0, x)=u_{o}(x) & &
\end{array}\right.
$$

is a map $u \in C^{0}\left([0, T] ; \bar{u}+L^{1}\left(\mathbb{R}^{+} ; \Omega^{n}\right)\right)$ such that for all $t \in[0, T], u(t) \in B V\left(\mathbb{R}^{+} ; \Omega^{n}\right)$ and

$(\mathbf{W}): u(0)=u_{o}$ and for all $\phi \in C_{c}^{\infty}(] 0, T\left[\times \mathbb{R}^{+} ; \mathbb{R}\right)$ and for $l=1, \ldots, n$

$$
\int_{0}^{T} \int_{\mathbb{R}^{+}}\left(u_{l} \partial_{t} \phi+f\left(u_{l}\right) \partial_{x} \phi\right) d x d t=0 .
$$

$(\Psi)$ : The condition at the junction is met: For a.e. $t \in \mathbb{R}^{+}, \Psi(u(t, 0+))=0$.

The weak solution $u$ is an entropy solution if for any convex entropy-entropy flux pair $\left(\eta_{l}, q_{l}\right)$, for all $\phi \in C_{c}^{\infty}(] 0, T\left[\times \mathbb{R}^{+} ; \mathbb{R}^{+}\right)$and for $l=1, \ldots, n$,

$$
\int_{0}^{T} \int_{\mathbb{R}^{+}}\left(\eta_{l}\left(u_{l}\right) \partial_{t} \phi+q_{l}\left(u_{l}\right) \partial_{x} \phi\right) d x d t \geq 0
$$

The main properties of a solution to the coupled problem are therefore as in a Cauchy problem. We have bounded variation, entropic solutions along arcs and the coupling condition is met a.e. in $t$.

Further, for a point $\hat{u}_{j}$ in the (close) vicinity of $\bar{u}_{j}$ we expect that the Jacobian of $f$, $A_{j}:=D f\left(\hat{u}_{j}\right)$, also admits a strictly negative $\lambda_{j, 1}$ and strictly positive eigenvalue $\lambda_{j, 2}$ with linearly independent (right) eigenvectors $r_{j, 1}$ and $r_{j, 2}$, respectively. The associated characteristic fields are assumed to be either genuine nonlinear or linearly degenerate. We will use this notation throughout the manuscript. The dependence of the eigenvectors 
and eigenvalues on $\hat{u}_{j}$ will be denoted by subindices as well as the corresponding fields $i=1,2$. A superindex $(\ell=1,2)$ will be used to denote the component $v^{\ell}$ of a vector $v \in \mathbb{R}^{2}$.

Now we present the finite volume method $[34,36]$ which is employed to numerically solve (1)-(3). This is essentially based on the proof of the well-posedness.

Consider a regular (uniform) grid of cell size $\Delta x=x_{i+\frac{1}{2}}-x_{i-\frac{1}{2}}$, where $x_{i-\frac{1}{2}}$ is the interface point (between cell $i-1$ and $i$, for $x_{i+\frac{1}{2}}=x_{i+1-\frac{1}{2}}$ analog) and time step $\Delta t=t^{m+1}-t^{m}$, chosen so that the CFL condition [19] $\lambda_{\max } \Delta t \leq \Delta x$, where $\lambda_{\max }$ is the largest wave speed, is satisfied. We assume the grid points are labeled by $i \in \mathbb{N}_{0}$ and the time steps by $m \in \mathbb{N}_{0}$ such that $x_{0}=\frac{\Delta x}{2}$ and $t^{0}=0$. Hence, the center of the first cell $i=0$ in each arc is located at $x_{0}$ and the physical node is located at the boundary $x=0$. For some compact domain $\Omega \subset \mathbb{R}^{2}$ such that $u_{j, 0} \in \Omega$ and $u_{j}(x, t) \in \Omega$, we compute the spectral radius $\rho$ of $D f$ as

$$
\lambda_{\max }=\max _{v \in \Omega}|\rho(D f(v))| .
$$

In addition the cell boundaries (interfaces) are denoted $x_{i-1 / 2}$, on the left, and $x_{i+1 / 2}$ on the right such that $\Delta x=x_{i+1 / 2}-x_{i-1 / 2}$. Sometimes the notation $\mathcal{I}_{i}:=\left[x_{i-1 / 2}, x_{i+1 / 2}\right]$ will be used. The discretization is undertaken component-wise for each $u_{j}$. Hence the cell average of $u_{j}$ in cell $i$ at time $t^{m}$ is denoted by $U_{j, i}^{m}$ and defined as

$$
U_{j, i}^{m}:=\frac{1}{\Delta x} \int_{x_{i-\frac{1}{2}}}^{x_{i+\frac{1}{2}}} u_{j}\left(x, t^{m}\right) d x .
$$

The evolution of the cell average over a single time-step, $\Delta t$, is

$$
U_{j, i}^{m+1}=U_{j, i}^{m}-\frac{1}{\Delta x}\left(\left(\mathcal{F}_{j}\right)_{i+\frac{1}{2}}-\left(\mathcal{F}_{j}\right)_{i-\frac{1}{2}}\right),
$$

where the numerical flux across the cell interface in $\operatorname{arc} j$ is given by

$$
\left(\mathcal{F}_{j}\right)_{i+\frac{1}{2}}=\int_{t^{m}}^{t^{m+1}} f\left(u_{j}\left(x_{i+\frac{1}{2}}, s\right)\right) d s .
$$

In Godunov's method [27] $\left(\mathcal{F}_{j}\right)_{i+\frac{1}{2}}=\mathcal{F}_{j}\left(U_{j, i}^{m}, U_{j, i+1}^{m}\right)$ in which the exact solution to a Riemann problem posed at the cell boundary $i+\frac{1}{2}$ is used to define the numerical flux $\left(\mathcal{F}_{j}\right)_{i+\frac{1}{2}}$. Thus in the original Godunov's method a piecewise constant reconstruction

$$
u_{j}\left(x, t^{m}\right)=\sum_{i} U_{j, i}^{m} \chi_{\left[x_{i-\frac{1}{2}}, x_{i+\frac{1}{2}}\right]}(x),
$$

where the characteristic function $\chi(x)$ is defined in the usual way as

$$
\chi_{\left[x_{i-\frac{1}{2}}, x_{i+\frac{1}{2}}\right]}(x)= \begin{cases}1, & \text { if } x \in\left[x_{i-\frac{1}{2}}, x_{i+\frac{1}{2}}\right] \\ 0, & \text { otherwise, }\end{cases}
$$

was applied as a numerical approximation for $u_{j}(x, t)$ at time $t^{m}$.

To generalize Godunov's method, the piecewise constant approximation (9) of the solution can be replaced by a more accurate representation. In this paper, we consider 
a piecewise linear approximation as e.g. employed in the MUSCL scheme [43]. Thus the reconstruction in (9) takes the form

$$
u_{j}\left(x, t^{m}\right)=\sum_{i} P_{i}\left(x, t^{m} ; U_{j, \cdot}^{m}\right) \chi_{\left[x_{i-\frac{1}{2}}, x_{i+\frac{1}{2}}\right]}(x),
$$

where $P_{i}\left(x, t^{m} ; U_{j, .}^{m}\right)$ is a linear reconstruction in cell $\mathcal{I}_{i}$ using data $\left\{U_{j, .}^{m}\right\}$ in arc $j$ such that

$$
P_{i}\left(x, t^{m} ; U_{j, .}^{m}\right)=U_{j, i}^{m}+\sigma_{j, i}^{m}\left(x-x_{i}\right),
$$

for $x \in \mathcal{I}_{i}$. The slope $\sigma_{j, i}^{m}$ in cell $\mathcal{I}_{i}$ is also based on the data $\left\{U_{j,}^{m}\right\}$. Note that $\sigma_{j, i}^{m}=0$ recovers the first-order Godunov method. Furthermore, the upcoming second order reconstruction of the coupling at the vertex is independent of the chosen scheme solving the dynamics at an non-boundary cell. The following algorithm does not dependent on the slope reconstruction and therefore any other finite-volume based method could be employed, for example WENO or discontinuous Galerkin methods.

A slope limiter is applied to $\sigma_{j, i}^{m}[38]$. Again, any slope limiter might be employed and a simple choice of slopes is the minmod slope limiter based on

$$
\sigma_{j, i}^{m}=\frac{1}{\Delta x} \operatorname{minmod}\left(U_{j, i+1}^{m}-U_{j, i}^{m}, U_{j, i}^{m}-U_{j, i-1}^{m}\right),
$$

where the minmod function is defined as:

$$
\begin{aligned}
\operatorname{minmod}(a, b) & = \begin{cases}a, & \text { if }|a|<|b| \text { and } a b>0 \\
b, & \text { if }|b|<|a| \text { and } a b>0 \\
0, & \text { if } a b \leq 0\end{cases} \\
& =\frac{1}{2}(\operatorname{sgn}(a)+\operatorname{sgn}(b)) \min (|a|,|b|) .
\end{aligned}
$$

It can be noted that, just as in first-order Godunov method, a Riemann problem needs to be solved at the cell boundaries $x_{i+1 / 2}$ since the reconstruction provides two values at the cell interfaces $x_{i+1 / 2}$ which we denote by

$$
u_{j}\left(x_{i+\frac{1}{2}}^{-}, t^{m}\right)=U_{j, i-}^{m}, \quad u_{j}\left(x_{i+\frac{1}{2}}^{+}, t^{m}\right)=U_{j, i+}^{m},
$$

which are values based on the polynomial $\left.P_{i}(x)\right|_{x=x_{i+\frac{1}{2}}}$ on the left of the interface and $\left.P_{i+1}(x)\right|_{x=x_{i+\frac{1}{2}}}$ on the right of the interface, respectively.

The coupling condition (3) at the vertex induces a boundary condition for equation (1) at $x=0$. At time $t^{m}$ the cell averages in the first cell $i=0$ of the connected arcs $j$ are given by $U_{j, 0}^{m}$ for all $\operatorname{arcs} j=1, \ldots, n$. Condition (4) ensures the local invertibility at a state $\left(\hat{u}_{j}\right)_{j=1}^{n}$. Due to the differentiability of $\Psi$ this property holds also in a neighborhood of this state. Due to the differentiability of $f$ also the sign of the eigenvalues $\lambda_{j, \ell}$ for $\ell=1,2$ do not change in this neighborhood. Therefore, we condition (4) holds for $\left(U_{j, 0}^{m}\right)_{j=1}^{n}$ provided $\left\|U_{j, 0}^{m}-\hat{u}_{j}\right\|$ is sufficiently small. Unfortunately, there is no rigorous estimate the difference available. However, in the numerical simulations we did not encounter a case where condition (4) is not met.

Denote the $\kappa$-th Lax curve through the state $u_{0}$ for $\kappa=1,2$. by $s \rightarrow \mathcal{L}_{\kappa}\left(u_{o}, s\right)$. The definition of a Lax curve can be found for example in [20]. In general, the Lax-curve through $u_{0}$ provides a parameterization of the admissible states that can be connected 
to $u_{0}$ by either a rarefaction wave or an Lax-admissible shock. Other entropy conditions are possible but we focus here, as in prior work, on the Lax-entropy condition [20]. Due to the requirement of $u_{0}$ being subsonic all states along the 2-Lax curve provide states such that the resulting Riemann problem admits waves of non-negative speed. We also refer to [7] for more details on the discussion of admissible Lax-curves and the solution to coupled problems.

Finally, solve for $\left(s_{1}^{*}, \ldots, s_{n}^{*}\right)$ using, for example, Newton's method the nonlinear system

$$
\Psi\left(\mathcal{L}_{2}\left(U_{1,0}^{m}, s_{1}\right), \ldots, \mathcal{L}_{2}\left(U_{n, 0}^{m}, s_{n}\right)\right)=0 .
$$

Note that a unique solution of (11) exists due to (4). The boundary value $U_{j, 0}^{m+1}$ at time $t^{m+1}$ is then given by equation (7) for $i=0$ and with

$$
U_{j,-1}^{m}:=\mathcal{L}_{2}\left(U_{j, 0}^{m}, s_{j}^{*}\right), j \in\{1, \ldots, n\} .
$$

This construction yields a first-order approximation to the coupling condition and the solution $u_{j}$.

In order to obtain at least a first-order convergent scheme, we consider a reconstruct, evolve and average algorithm [36]. The finite-volume formulation of equation (1) is given by equation (7) and equation (8). We proceed as follows:

STEP1 Given the cell averages $U_{j, i}^{m}$, we reconstruct a piecewise linear function $u_{j}\left(x, t^{m}\right)$ on each cell $\left[x_{i-\frac{1}{2}}, x_{i+\frac{1}{2}}\right]$. This is a standard procedure and more details can be found, for example, in $[32,43]$. We apply a reconstruction with slopes obtained by the minmod limiter for the cells $i=1, \ldots$, as discussed for equation (10) above.

In the first cell $i=0$, we modify the reconstruction of the slope due to the absence of cell averages beyond the vertex since

$$
\sigma_{j, 0}^{m}=\frac{1}{\Delta x} \operatorname{minmod}\left(U_{j, 1}^{m}-U_{j, 0}^{m}, U_{j, 0}^{m}-U_{j,-1}^{m}\right) .
$$

Instead, for $U_{j,-1}^{m}$ the procedure applied for the first-order case can be applied here, see equation (12).

Denote the vector of the recovered slopes for both components by $\sigma_{j, i}=$ $\left(\sigma_{j, i}^{1}, \sigma_{j, i}^{2}\right)$ for $i>0$. Using the above construction, we obtain the reconstruction

$$
u_{j}\left(x, t^{m}\right)=\sigma_{j, i}\left(x-x_{i}\right)+U_{j, i}^{m}, \quad x_{i-\frac{1}{2}} \leq x \leq x_{i+\frac{1}{2}}, i=1, \ldots
$$

The previous reconstruction provides two values at the cell interfaces $x_{i+\frac{1}{2}}$ denoted in the following by $x_{i+\frac{1}{2}} \mp$ for the reconstruction at $x_{I+\frac{1}{2}}$ from cell $i$ and $i+1$, respectively.

STEP2 Reconstruct for each arc $j$ a piecewise linear function $v_{j}(t)$ for $t^{m} \leq t \leq t^{m+1}$ such that

$$
\Psi\left(v_{1}\left(t^{m}\right), \ldots, v_{n}\left(t^{m}\right)\right)=0 .
$$


and

$$
\left.\frac{d}{d t} \Psi\left(v_{1}(t), \ldots, v_{n}(t)\right)\right|_{t=t^{m}}=0
$$

STEP3 Evaluate equation (8) using a predictor-corrector step [37] for all cells except for $i=0$. This approach is also attributed to Hancock in $[42,43]$. We split the flux as in a Lax-Friedrichs scheme

$$
f(u)=f^{+}(u)+f^{-}(u):=\frac{1}{2}(f(u)+a u)+\frac{1}{2}(f(u)-a u),
$$

where $a=\lambda_{\max }$. Due to splitting the wave speeds of the fluxes $f(u) \pm a u$ do not change sign across $x_{i+\frac{1}{2}}$. Using this fact and the midpoint rule at time $t^{m+\frac{1}{2}}=$ $t^{m}+\frac{\Delta t}{2}$, the evolution of the flux (8) is given by

$$
\left(\mathcal{F}_{j}\right)_{i+\frac{1}{2}}=\Delta t\left(f ^ { + } \left(u_{j}\left(x_{i+\frac{1}{2}}-, t^{m+\frac{1}{2}}\right)+f^{-}\left(u_{j}\left(x_{i+\frac{1}{2}}+, t^{m+\frac{1}{2}}\right)\right)+O\left((\Delta t)^{3}\right) .\right.\right.
$$

Using Taylor expansion and the linear reconstruction (13), we obtain up to second order in space and time

$$
\begin{aligned}
& U_{j, i-}^{m}:=U_{j, i}^{m}+\sigma_{j, i} \frac{\Delta x}{2}, U_{j, i+}^{m}:=U_{j, i+1}^{m}-\sigma_{j, i+1} \frac{\Delta x}{2} \\
& \frac{1}{\Delta x}\left(\mathcal{F}_{j}\right)_{i+\frac{1}{2}} \approx \frac{\Delta t}{\Delta x}\left(f^{+}\left(U_{j, i-}^{m}-\frac{\Delta t}{2} D f\left(U_{j, i-}^{m}\right) \sigma_{j, i}\right)+\right. \\
& \left.f^{-}\left(U_{j, i+}^{m}-\frac{\Delta t}{2} D f\left(U_{j, i+}^{m}\right) \sigma_{j, i+1}\right)\right) .
\end{aligned}
$$

STEP4 Evaluate the fluxes in cell $i=0$. The only flux which needs to be evaluated is $\left(\mathcal{F}_{j}\right)_{-\frac{1}{2}}$. Due to the construction of the boundary conditions in STEP2 the arising waves will have non-negative wave speed. Therefore,

$$
\left(\mathcal{F}_{j}\right)_{-\frac{1}{2}}=\int_{t^{m}}^{t^{m+1}} f\left(u_{j}\left(x_{-\frac{1}{2}}, s\right)\right) d s \approx \int_{t^{m}}^{t^{m+1}} f\left(v_{j}(s)\right) d s=\Delta t f\left(v_{j}\left(t^{m+\frac{1}{2}}\right)\right)+O\left((\Delta t)^{3}\right) .
$$

Similarly, to STEP3 we evaluate

$$
\frac{1}{\Delta x}\left(\mathcal{F}_{j}\right)_{-\frac{1}{2}} \approx \frac{\Delta t}{\Delta x} f\left(v_{j}\left(t^{m}\right)+\left.\frac{\Delta t}{2} \frac{d}{d t} v_{j}(t)\right|_{t=t^{m}}\right) .
$$

STEP5 Evolve the dynamics according to equation ( 7$)$ for $i=1, \ldots$, to obtain the new cell averages at time $t^{m+1}$ and proceed with STEP1.

To approximate the slopes $\sigma_{j, 0}$ we first calculate the piecewise constant information $U_{j,-1}$ as given by (12). This data can now be used to gain the $\sigma_{j, 0}$ in the same way as in STEP1. Currently, there is no estimate that the construction STEP2 to STEP4 will also guarantee the TVD property known for high-order finite volume schemes.

The difference to a standard first-order method is STEP2 and STEP3. In order to determine the values $v_{j}\left(t^{m}\right)$, we proceed similarly to the discussion in equation (11). 
However, since we reconstruct the function $u_{j}\left(x, t^{m}\right)$ on arc $j$ by a piecewise linear function, the value of $u_{j}\left(x, t^{m}\right)$ closest to the vertex at time $t^{m}$ is given by

$$
u_{j}\left(x_{-\frac{1}{2}}, t^{m}\right)=U_{j, 0}^{m}-\frac{\Delta x}{2} \sigma_{j, 0} .
$$

Hence, we determine the vector $s=\left(s_{1}, \ldots, s_{n}\right) \in \mathbb{R}^{n}$ by solving the possibly nonlinear equation (15).

$$
\Psi\left(\mathcal{L}_{2}\left(U_{1,0}^{m}-\frac{\Delta x}{2} \sigma_{1,0}, s_{1}\right), \ldots, \mathcal{L}_{2}\left(U_{n, 0}^{m}-\frac{\Delta x}{2} \sigma_{n, 0}, s_{n}\right)\right)=0
$$

For $U_{j, 0}^{m}$ sufficiently close to $\hat{u}$ and due to condition (4), the previous equation has a unique solution $s$. Hence we define

$$
v_{j}\left(t^{m}\right):=\mathcal{L}_{2}\left(U_{j, 0}^{m}-\frac{\Delta x}{2} \sigma_{j, 0}, s_{j}\right)
$$

For the reconstruction in equation (14), we additionally need at least to recover also the slope $\frac{d}{d t} v_{j}(t)$ at time $t=t^{m}$. Condition (3) is supposed to hold true also for $t \geq t^{m}$. Hence, we obtain for sufficiently smooth solutions

$$
0=\frac{d}{d t} \Psi\left(u_{1}(0+, t), \ldots, u_{n}(0+, t)\right)=\sum_{k=1}^{n} D_{u_{k}} \Psi\left(u_{1}(0+, t), \ldots, u_{n}(0+, t)\right) \partial_{t} u_{k}(0+, t) .
$$

Note that in in a neighborhood of the state $\hat{u}$ we have $\lambda_{1}\left(u_{k}(0+, t)\right)<0<\lambda_{2}\left(u_{k}(0+, t)\right)$. Therefore, we expect that only a part of the information of $\partial_{t} u_{k}(0+, t)$ is available at the vertex. Further, due to (16) we will have waves emerging from the vertex in short time. Therefore, in of $D_{u_{k}} \Psi\left(u_{1}(0+, t), \ldots, u_{n}(0+, t)\right)$ we have $u_{k}(0+, t)=v_{k}(t)$ for $t>t^{m}$. We now approximate $\partial_{t} u_{k}(0+, t)$ for $t>t^{m}$ using a Roe-type scheme and compute the derivative $A_{j}$ of $f$ at the new position $v_{j}\left(t^{m}\right)$. Let the constant matrix $A_{j}$ be defined by

$$
A_{j}:=\operatorname{Df}\left(v_{j}\left(t^{m}\right)\right), j=1, \ldots, n .
$$

Since $f$ is strictly hyperbolic and for $v_{j}\left(t^{m}\right)$ in the neighborhood of $\hat{u}$, each $A_{j}$ is diagonalizable with eigenvalues $\lambda_{j, 1}<0$ and $\lambda_{j, 2}>0$ and corresponding linearly independent set of eigenvectors $r_{j, 1}=r_{1}\left(v_{j}\left(t^{m}\right)\right)$ and $r_{j, 2}=r_{2}\left(v_{j}\left(t^{m}\right)\right)$, respectively. Let $R_{j}:=\left(r_{j, 1}, r_{j, 2}\right)$. For small values of $t-t^{m} \geq 0$, we approximate $u_{j}(0+, t)$ by a decomposition in eigenvectors

$$
u_{j}(t, 0+) \approx \mathbf{v}_{j}^{1}(t) r_{j, 1}+\mathbf{v}_{j}^{2}(t) r_{j, 2}=: R_{j}\left(\begin{array}{c}
\mathbf{v}_{j}^{1}(t) \\
\mathbf{v}_{j}^{2}(t)
\end{array}\right)
$$

Further, we approximate the dynamics of $\partial_{t} \mathbf{v}_{j, 1}(t)$ for small $t-t^{m}$ by

$$
\partial_{t} \mathbf{v}_{j}^{1}\left(t^{m}\right)=-\lambda_{j}^{1} \partial_{x}\left(R_{j}^{-1} u_{j}\left(t^{m}, 0+\right)\right)^{1}=-\lambda_{j}^{1}\left(R_{j}^{-1}\left(\begin{array}{c}
\sigma_{j, 0}^{1} \\
\sigma_{j, 0}^{2}
\end{array}\right)\right)^{1} .
$$

where we used the linear reconstruction of $u_{j}(x, t)$ on arc $j$ and the super-index denotes the component $\in\{1,2\}$ of the vector. Then, we obtain from equation (17) at time $t^{m}$ 
an equation in the unknowns $\mathbf{v}_{j}^{2}$

$$
0=\sum_{k=1}^{n} D_{k} \Psi\left(v_{1}\left(t^{m}\right), \ldots, v_{n}\left(t^{m}\right)\right) R_{j}\left(\begin{array}{c}
\mathbf{v}_{j}^{1}(t) \\
\mathbf{v}_{j}^{2}(t)
\end{array}\right)
$$

or equivalently

$$
\begin{array}{r}
\sum_{k=1}^{n} D_{k} \Psi\left(v_{1}\left(t^{m}\right), \ldots, v_{n}\left(t^{m}\right)\right) r_{1}\left(v_{k}\left(t^{m}\right) \lambda_{k}^{1}\left(R_{k}^{-1}\left(\begin{array}{c}
\sigma_{k, 0}^{1} \\
\sigma_{k, 0}^{2}
\end{array}\right)\right)^{1}=\right. \\
\sum_{k=1}^{n} D_{k} \Psi\left(v_{1}\left(t^{m}\right), \ldots, v_{n}\left(t^{m}\right) r_{2}\left(v_{k}\left(t^{m}\right) \partial_{t} \mathbf{v}_{k}^{2}\left(t^{m}\right) .\right.\right.
\end{array}
$$

Again, due to condition (4) the previous equation (20) has a unique solution for the $n$ values $\partial_{t} \mathbf{v}_{j}^{2}\left(t^{m}\right), j=1, \ldots, n$. Then, we set

$$
\frac{d}{d t} v_{j}\left(t^{m}\right):=\sum_{\ell=1}^{2} \partial_{t} \mathbf{v}_{j}^{\ell}\left(t^{m}\right) r_{\ell}\left(v_{j}\left(t^{m}\right)\right) .
$$

A piecewise linear reconstruction of $v_{j}$ yields at time $t=t^{m}$

$$
\Psi\left(v_{1}\left(t^{m}\right)+\frac{d}{d t} v_{1}\left(t^{m}\right)\left(t-t^{m}\right), \ldots, v_{n}\left(t^{m}\right)+\frac{d}{d t} v_{n}\left(t^{m}\right)\left(t-t^{m}\right)\right)=O\left(\left(t-t^{m}\right)^{2}\right) .
$$

We summarize the computations in the following Lemma.

Lemma 2.3. Consider a single node with $n$ connected arcs and let $t^{m}$ be some positive time. Let $\Psi \in C^{2}\left(\mathbb{R}^{2 n} ; \mathbb{R}^{n}\right)$ and let $\hat{u}_{j}:=U_{j, 0}^{m}-\frac{\Delta x}{2}$ be such that equation (4) holds true. Then, for $v_{j}(t)$ as in the previous construction, the coupling condition is satisfied up to second order in time, i.e.

$$
\Psi\left(v_{1}(t), \ldots, v_{n}(t)\right)=O\left(\left(t-t^{m}\right)^{2}\right) .
$$

Remark 2.4. Note that the construction is similar to the work [39]. Therein, this procedure has been applied to obtain high-order boundary values. The only difference lies in the fact that we do not have the boundary values at hand. Those are only given implicitly through the coupling condition $\Psi$ and are obtained through solving a nonlinear system.

Remark 2.5. The current procedure has been presented for $2 \times 2$ conservation laws with states in the vicinity of a subsonic initial state $U_{i, 0}$ and $i=1, \ldots, n$. In the case of $2 \times 2$ conservation laws this leaves one degree of freedom for each attached arc $i$ and therefore $\Psi(\cdot) \in \mathbb{R}^{n}$. An extension of the procedure to $k \times k$ conservation laws is possible using the same ideas. However, in general, more coupling conditions will be required. In the $k \times k$ case we have for each Riemann problem at most $k$ waves emerging. The number of conditions dependent on the initial data $U_{i, 0} \in \mathbb{R}^{k}$. More precisely, let for each $i$ in the vicinity of the initial data $U_{i, 0} \in \mathbb{R}^{k}$ be $\mathcal{U}_{i}(\ell \leq k)$ an $\ell=\ell(i)$ dimensional subspace such that for each $V_{i} \in \mathcal{U}_{i}$ the solution to the Riemann problem with initial data $U_{l}=V_{i}$ and $U_{r}=U_{i, 0}$ allows for non-negative waves. Then, we require at least $L$ conditions, i.e., $\Psi \in C^{1}\left(\mathbb{R}^{n k} ; \mathbb{R}^{L}\right)$ where $L:=\sum_{i=1}^{n} \ell(i)$. Further, we require a transversality condition similar to (4) in order to ensure local invertibility. 
Besides the (simple) example of the linear case we are only aware of one reference for a $3 \times 3$ system where this procedure has been discussed [18].

Remark 2.6. An extension to higher than second-order is also possible. In this case STEP2 has to be modified and higher temporal derivatives of $\Psi$ need to be considered. Again, condition (4) will ensure invertibility of the linearized coupling conditions. Further, higher derivatives of the flux $f$ have to be considered in order to obtain the highorder reconstruction of the state at the node. It is planned to study this case in future work.

\section{Properties of the SCheme in the Linear CASE}

In order to illustrate the properties of the scheme, we first consider the case of a single arc extending from $-\infty$ to $\infty$. We drop for a moment in the index $j$. Also, assume that $u \in \mathbb{R}, f: \mathbb{R} \rightarrow \mathbb{R}$ and is given by

$$
f(u)=c u, c>0 .
$$

Remark 3.1. We are aware, that this setting does not fit in the assuptions. Nevertheless represents the scalar advection case exactly our procedure applied on a linear $2 \times 2$ system that holds all postulated assumptions. Since the scaler advection is the transport within each charactersitic of the linear system.

Since $a=c$, we obtain $f^{+}(u)=c u, f^{-}(u)=0$ and $D f^{ \pm}(u)=\frac{1}{2}(c \pm a)$. The CFL condition gives $c \Delta t \leq \Delta x$. The numerical flux at the cell boundary is then given by

$$
\mathcal{F}_{i+\frac{1}{2}}=\frac{\Delta t}{\Delta x} c\left(U_{i}^{m}+\sigma_{i} \frac{\Delta x}{2}-\frac{c \Delta t}{2} \sigma_{i}\right) .
$$

The scheme, therefore, takes the form:

$$
U_{i}^{m+1}=U_{i}^{m}-\frac{\Delta t}{\Delta x} c\left(U_{i}^{m}-U_{i-1}^{m}+\frac{\Delta x-c \Delta t}{2}\left(\sigma_{i}-\sigma_{i-1}\right)\right) .
$$

One easily verifies that this method is second order accurate in space and time.

Further, we consider the scalar case $n=1$ and two connected $\operatorname{arcs} j=1,2$ with $f_{1}(u)=-c u, f_{2}(u)=c u$ and $c>0$. Note that in this case we may not apply the previous construction directly. In order to have consistency with the Cauchy problem we use as coupling condition $\Psi$

$$
\Psi\left(u_{1}(0+, t), u_{2}(0+, t)\right)=u_{2}(0+, t)-u_{1}(0+, t)=0 .
$$

By transformation in $x$ the problem

$$
\partial_{t} u_{j}+\partial_{x} f_{j}\left(u_{j}\right)=0, x \geq 0, t \geq 0, \text { and } u_{2}(0+, t)-u_{1}(0+, t)=0, t \geq 0 .
$$

is equivalent to a Cauchy problem for $y(x, t)$ with combined domain $x \in \mathbb{R}$ and $t \geq 0$

$$
\partial_{t} y(x, t)+c \partial_{x} y(x, t)=0, y(x, 0)=y_{0}(x):=\left(\begin{array}{cc}
u_{1,0}(-x), & \text { for } x \leq 0 \\
u_{2,0}(x), & \text { for } x>0 .
\end{array}\right) .
$$

In the following derivations, we show that the previous construction leads to a scheme of second order (24) in space and time for equation (27). Clearly, the eigenvalues are $\lambda=-c$ and $\lambda=c$ for arcs $j=1,2$, respectively, and therefore for arc $j=1$ the Laxcurve is a constant given by the state $\mathcal{L}_{2}\left(\hat{u}_{1}, s_{1}\right)=\hat{u}_{1}$. Hence, we do not prescribe any 
boundary condition for $\operatorname{arc} j=1$. In order to use a similar notation as above, we note that the admissible boundary states for arc $j=2$ are given by

$$
\mathcal{L}_{2}\left(\hat{u}_{2}, s_{2}\right)=s_{2}+\hat{u}_{2}, s_{2} \in \mathbb{R} .
$$

In $\operatorname{arc} j=1$, we have $\mathcal{L}_{2}\left(\hat{u}_{1}, s_{1}\right)=\hat{u}_{1}$. Hence, for the unknown $s_{2}$ the condition (15) becomes:

$$
U_{1,0}^{m}-\frac{\Delta x}{2} \sigma_{1,0}-\left(U_{2,0}^{m}-\frac{\Delta x}{2} \sigma_{2,0}+s_{2}\right)=0 .
$$

and equation (16) yields:

$$
v_{2}\left(t^{m}\right)=U_{1,0}^{m}-\frac{\Delta x}{2} \sigma_{1,0}, v_{1}\left(t^{m}\right)=U_{1,0}^{m}-\frac{\Delta x}{2} \sigma_{1,0} .
$$

Repeating the computations (17) to (22) we obtain, for sufficiently smooth solution $u_{1}(0+, t)$,

$$
0=\partial_{t} u_{1}(0+, t)-\partial_{t} u_{2}(0+, t)=+c \partial_{x} u_{1}(0+, t)-R_{2}=c \sigma_{1,0}-R_{2} .
$$

Here, we also used the fact that on arc $j=1$ and for sufficiently smooth $u_{1}$, we have $\partial_{t} u_{1}(0+, t)-c \partial_{x} u_{1}(0+, t)=0$. Therefore, the linear reconstruction of $v_{2}(t)$ for $t^{m+1}>$ $t \geq t^{m}$ reads

$$
v_{2}(t)=U_{1,0}^{m}-\frac{\Delta x}{2} \sigma_{1,0}+c \sigma_{1,0}\left(t-t^{m}\right) .
$$

The numerical flux in cell $i=0$ is then given as

$$
\left(\mathcal{F}_{2}\right)_{-\frac{1}{2}}=\Delta t c\left(U_{1,0}^{m}-\frac{\Delta x}{2} \sigma_{1,0}+\frac{\Delta t}{2} c \sigma_{1,0}\right) .
$$

In order to compare the proposed method with a numerical discretization of equation (27) it suffices to consider the discretization of the first cell $i=0$ of arc $j=2$. The remaining cells are independent of the coupling condition and therefore the applied discretization coincide. In the first cell $i=0$ of arc $j=2$ we obtain from (30) and (14)

$$
U_{2,0}^{m+1}=U_{2,0}^{m}-\frac{\Delta t}{\Delta x} c\left(U_{2,0}^{m}-U_{1,0}^{m}+\left(\sigma_{2,0}+\sigma_{1,0}\right) \frac{\Delta x-c \Delta t}{2}\right) .
$$

Suppose a discretization of equation (27) with $i \in \mathbb{N}$ and such that at $x=0$ we have the location of the physical node and as before $x_{i}=\frac{\Delta x}{2}$ for $i=0$ is the location of the center of the grid. The discretization (31) has to be compared with a corresponding second order discretization of equation (27) in cell $i=0$, i.e., for $x \in\left[-\frac{\Delta x}{2}, \frac{\Delta x}{2}\right]$. Using equation (24) we obtain for the cell averages $Y_{i}^{m}=\frac{1}{\Delta x} \int_{x_{i-\frac{1}{2}}}^{x_{i+\frac{1}{2}}} y\left(x, t^{m}\right) d x$

$$
Y_{0}^{m+1}=Y_{0}^{m}-\frac{\Delta t}{\Delta x} c\left(Y_{0}^{m}-Y_{-1}^{m}+\left(\tau_{0}-\tau_{-1}\right) \frac{\Delta x-c \Delta t}{2}\right) .
$$

The construction of $\sigma_{2,0}$ and $\tau_{0}$ are independent of the coupling condition and therefore $\sigma_{2,0}=\tau_{0}$. In the continuous case we have $y(-x, t)=u_{1}(x, t)$ and therefore $Y_{i}^{m}=U_{1,-i+1}^{m}$ for $i<0$ and the slopes of the linear reconstruction of $y$ and $u_{1}$ are related as $\sigma_{1,-i}=$ $-\tau_{i+1}, i<0$. Hence, provided we use the same derivation of the slopes for $U_{j, i}$ and $Y_{i}$ we observe that the proposed discretization of the coupling condition leads to the same 
scheme as a discretization for the Cauchy problem (27). In particular, the coupling condition does not lead to a reduction of reduction the scheme. The same construction with opposite roles for arcs one and two yields then the case $c<0$.

Remark 3.2. The construction would even allow equation (23) for the case $c=c_{+} H(x)+$ $c_{-} H(-x)$ with $c_{+}>0$ and $c_{-}<0$. Here, $H(x)$ is the Heavi-side function. However in this case a single coupling condition is not sufficient to obtain a well-posed problem.

We summarize the findings in the following Lemma.

Lemma 3.3. Let $n=2$ and consider for $(x, t) \in \mathbb{R}_{0}^{+} \times \mathbb{R}^{+}$the problem

$$
\partial_{t} u_{1}+c \partial_{x} u_{1}=0, \partial_{t} u_{2}-c \partial_{x} u_{2}=0
$$

with initial data given by (2) and coupling condition (25). On an equidistant spatial grid $\left(x_{i}\right), i \in \mathbb{N}$ consider a piecewise linear reconstruction $u_{j}\left(x, t^{m}\right)$ for $j=1,2$. Consider furthermore the second-order MUSCL discretization (32) of equation (27) with initial data at time $t=t^{m}$ given by $y\left(x, t^{m}\right)=u_{j}\left(-x, t^{m}\right) H(-x)+u_{j}\left(x, t^{m}\right) H(x)$.

Then, for $c=a$ and $j \geq 0$, the cell averages $U_{1, j}^{m+1}$ and $U_{2, j}^{m+1}$ obtained from the proposed algorithm coincide with the cell averages $Y_{-j-1}^{m+1}$ and $Y_{j}^{m+1}$, respectively.

Next, we consider the case of a linear system $f(u)=A u$ with $A \in \mathbb{R}^{2}$ strictly hyperbolic with $\lambda_{1}<0$ and $\lambda_{2}>0$. We denote by $R=\left(r_{1}, r_{2}\right)$ the matrix of the (right) eigenvectors to $A$. Further, consider the case of a single arc $n=1$. Then, problem (1)(3) is a boundary value problem for $u_{1}(x, t)$ with $x \geq 0$ and boundary conditions induced by equation (3). We prescribe as boundary condition $t \rightarrow b(t), b \in C^{1}$, in the second characteristic variable. In terms of $\Psi$, we obtain

$$
\Psi\left(u_{1}(0+, t)\right)=\left(R^{-1} u_{1}(0+, t)\right)^{2}-b(t) .
$$

On the time interval $\left(t^{m}, t^{m+1}\right)$ we expect the linear construction in the second component $v_{1}(t) \in \mathbb{R}^{2}$ to be

$v(t)=R\left(\left(R^{-1}\left(u_{1}\left(0+, t^{m}\right)+\partial_{t} u_{1}\left(0+, t^{m}\right)\right)\right)^{1}, b\left(t^{m}\right)+b^{\prime}\left(t^{m}\right)\left(t-t^{m}\right)\right)^{T}+O\left(\left(t-t^{m}\right)^{2}\right)$.

We compare the presented approach to the approach presented in [6]. Due to the linearity of $A$, we have for any $\hat{u} \in \mathbb{R}^{2}$

$$
\mathcal{L}_{2}(\hat{u}, s)=\hat{u}+s r_{2}
$$

At first we discuss the reconstruction of STEP2. Due to the linear reconstruction in each cell, we have $U_{1,0}^{m}-\frac{\Delta x}{2} \sigma_{1,0}=u_{1}\left(0+, t^{m}\right)+O(\Delta x)^{2}$. Equation (15), (16) and (33) 
yield

$$
\begin{aligned}
& 0=\Psi\left(\mathcal{L}_{2}\left(U_{1,0}^{m}-\frac{\Delta x}{2} \sigma_{1,0}, s_{1}\right)\right)=\left(R^{-1}\left(U_{1,0}^{m}-\frac{\Delta x}{2} \sigma_{1,0}+s_{1} r_{2}\right)\right)^{2}-b\left(t^{m}\right) \\
&=\left(R^{-1}\left(U_{1,0}^{m}-\frac{\Delta x}{2} \sigma_{1,0}\right)\right)^{2}+s_{1}-b(t) \\
& v_{1}\left(t^{m}\right)=U_{1,0}^{m}-\frac{\Delta x}{2} \sigma_{1,0}+s_{1} r_{2} \\
&=R\left(R^{-1}\left(U_{1,0}^{m}-\frac{\Delta x}{2} \sigma_{1,0}\right)\right)+b\left(t^{m}\right) r_{2}-\left(R^{-1}\left(U_{1,0}^{m}-\frac{\Delta x}{2} \sigma_{1,0}\right)\right)^{2} R\left(\begin{array}{c}
0 \\
1
\end{array}\right) \\
&=R\left(R^{-1}\left(U_{1,0}^{m}-\frac{\Delta x}{2} \sigma_{1,0}\right)-\left(R^{-1}\left(U_{1,0}^{m}-\frac{\Delta x}{2} \sigma_{1,0}\right)\right)^{2}\left(\begin{array}{c}
0 \\
1
\end{array}\right)\right)+R\left(\begin{array}{c}
0 \\
b\left(t^{m}\right)
\end{array}\right) \\
&=R\left(\left(R^{-1} u_{1}\left(0+, t^{m}\right)\right)^{1}+O(\Delta x)^{2}\right) . \\
& b\left(t^{m}\right)
\end{aligned}
$$

The slope $\frac{d}{d t} v_{1}\left(t^{m}\right)$ is computed using equation (17) to (22). Equation (17) reads

$$
0=\left(R^{-1} \partial_{t} u_{1}(0+, t)\right)^{2}-b^{\prime}(t),
$$

and we approximate $\partial_{t} u_{1}(0+, t)$ by decomposition in characteristic variables to obtain

$$
\partial_{t} \mathbf{v}_{1}^{1}\left(t^{m}\right)=-\lambda^{1}\left(R^{-1} \sigma_{1,0}\right)^{1}=\left(R^{-1} \partial_{t} u_{1}\left(0+, t^{m}\right)\right)^{1}+O(\Delta x)^{2} .
$$

and according to $(20)$ at time $t^{m}$

$$
\begin{aligned}
0 & =\left(R^{-1}\left(\left(R^{-1} \partial_{t} u_{1}\left(0+, t^{m}\right)\right)^{1} r_{1}+\partial_{t} \mathbf{v}_{1}^{2}\left(t^{m}\right) r_{2}\right)\right)^{2}-b^{\prime}\left(t^{m}\right) \\
& =\partial_{t} \mathbf{v}_{1}^{2}\left(t^{m}\right)-b^{\prime}\left(t^{m}\right) .
\end{aligned}
$$

Due to (22), we obtain for $t-t^{m}=O(\Delta x)$ the reconstruction

$$
\begin{aligned}
v_{1}(t) & =R\left(\begin{array}{c}
\left(R^{-1} u_{1}\left(0+, t^{m}\right)\right)^{1} \\
b\left(t^{m}\right)
\end{array}\right)+\left(t-t^{m}\right)\left(b^{\prime}\left(t^{m}\right) r_{2}+\left(R^{-1} \partial_{t} u_{1}\left(0+, t^{m}\right)\right)^{1} r_{1}\right) \\
& =R\left(\begin{array}{c}
\left.\left(R^{-1}\left(u_{1}\left(0+, t^{m}\right)\right)^{1}+\left(t-t^{m}\right) \partial_{t} u_{1}\left(0+, t^{m}\right)\right)\right)^{1}+O(\Delta x)^{2} \\
b\left(t^{m}\right)+\left(t-t^{m}\right) b^{\prime}\left(t^{m}\right)
\end{array}\right)
\end{aligned}
$$

In [6] the following approach has been proposed to obtain a high order reconstruction of $v_{1}(t)$. We apply this procedure to a first-order scheme studied herein. The derivation of $v_{1}\left(t^{m}\right)$ is as above. However, there is a difference in the approximation of the derivative $\partial_{t} v_{1}\left(t^{m}\right)$. Instead of a characteristic decomposition, the value of the derivative is approximated using the Lax-curve to the linearized system, i.e.,

$$
\mathcal{L}_{2}\left(\partial_{t} u_{1}(0+, t), s_{1}^{\prime}(t)\right)=\partial_{t} u_{1}(0+, t)+s_{1}^{\prime}(t) r_{2},
$$

and the (real valued) unknown $s_{1}^{\prime}(t)$ at time $t=t^{m}$ is obtained as solution to equation (35). In order to evaluate $\partial_{t} u_{1}(0+, t)$ the linearized equation, i.e., $\partial_{t} u_{1}\left(0+, t^{m}\right)=$ 
- $\left(\begin{array}{cc}\lambda^{1} & 0 \\ 0 & \lambda^{2}\end{array}\right) \sigma_{1,0}$ is used leading to an error of order $O(\Delta x)^{2}$ due to the spatial reconstruction of $u_{1}(x, t)$. Hence,

$$
0=\left(R^{-1} \partial_{t} u_{1}(0+, t)\right)^{2}-b^{\prime}\left(t^{m}\right)+s_{1}^{\prime}\left(t^{m}\right)\left(R^{-1} r_{2}\right)^{2}+O(\Delta x)^{2} .
$$

Finally, the reconstruction of $v_{1}(t)$ up to second order in time is given by

$$
\begin{aligned}
v_{1}(t) & =R\left(\begin{array}{c}
\left(R^{-1} u_{1}\left(0+, t^{m}\right)\right)^{1} \\
b\left(t^{m}\right)
\end{array}\right)+\left(t-t^{m}\right) \mathcal{L}_{2}\left(\partial_{t} u_{1}(0+, t), s_{1}^{\prime}(t)\right) \\
& =R\left(\begin{array}{c}
\left(R^{-1}\left(u_{1}\left(0+, t^{m}\right)+\left(t-t^{m}\right) \partial_{t} u_{1}\left(0+, t^{m}\right)\right)\right)^{1}+O(\Delta x)^{2} \\
b\left(t^{m}\right)+b^{\prime}\left(t^{m}\right)\left(t-t^{m}\right)+O(\Delta x)^{2}
\end{array}\right) .
\end{aligned}
$$

In characteristic variables $R v_{1}(t)$, the difference of (36) and (37) is of order $O(\Delta x)^{2}$ in the recovered boundary condition. This is an error of the order of the scheme. However, in the presented approach no information on $\left(R \partial_{t} u_{1}(0+, t)\right)^{2}$ (being the outgoing characteristic) is needed and for small $\Delta t$ the resolution of $b(t)$ is of order $\frac{\Delta t^{2}}{2}$ instead of order $(\Delta x)^{2}=\lambda_{\max }^{2}(\Delta t)^{2}$.

The previous relation also holds true in a more general setting: Consider $n$ connected arcs and denote the state at $x=0+$ on arc $k$ by $\hat{u}_{k}=U_{k, 0}^{m}-\frac{\Delta x}{2} \sigma_{k, 0}$. Denote by $A_{k}=$ $D f\left(\hat{u}_{k}\right)$ and by $R_{k}=\left(r_{k, 1}, r_{k, 2}\right)$ the matrix of (right) eigenvectors of $A_{k}$. The superscript in $u^{\ell}$ denotes the component $\ell$ of the respective vector $u$. Let $\hat{u}_{k, t}$ be $\hat{u}_{k, t}:=-A \sigma_{k, 0}=$ $\partial_{t} \hat{u}_{k}\left(0+, t^{m}\right)+O(\Delta x)^{2}$ and let $\Psi: \mathbb{R}^{2 n} \rightarrow \mathbb{R}^{n}$ be a $C^{1}$ function with partial derivatives $\Psi_{u_{k}}:=D_{u_{k}} \Psi(\hat{u})$. Then, [6] requires to solve the following system for $\xi=\left(\xi_{k}\right)_{k} \in \mathbb{R}^{n}$ and reconstruct $v(t) \in \mathbb{R}^{n}$ by

$$
\begin{aligned}
0 & =\sum_{k=1}^{n} \Psi_{u_{k}}\left(\hat{u}_{k, t}+\xi_{k} r_{k, 2}\right), \\
\frac{d}{d t} v_{k}\left(t^{m}\right) & :=\hat{u}_{k, t}+\xi_{k} r_{k, 2} .
\end{aligned}
$$

Due to condition (4) the matrix $\mathcal{A} \in \mathbb{R}^{n \times n}$ defined by

$$
\mathcal{A}=\left(\Psi_{u_{1}} r_{1,2}, \ldots, \Psi_{u_{n}} r_{n, 2}\right)
$$


is invertible and we obtain for the second component of $\frac{d}{d t} v_{j}\left(t^{m}\right)$ and $\xi_{j}=\left(\sum_{k=1}^{n} \mathcal{A}^{-1} \Psi_{u_{k}} \hat{u}_{k, t}\right)^{j}$ the following equivalence

$$
\begin{aligned}
\left(R_{j}^{-1} \frac{d}{d t} v_{j}\left(t^{m}\right)\right)^{2}= & \left(R_{j}^{-1} \hat{u}_{j, t}\right)^{2}-\xi_{j}\left(R_{j}^{-1} r_{j, 2}\right)^{2} \\
= & \left(R_{j}^{-1} \hat{u}_{j, t}\right)^{2}-\left(\sum_{k=1}^{n} \mathcal{A}^{-1} \Psi_{u_{k}} R_{k} R_{k}^{-1} \hat{u}_{k, t}\right)^{j} \\
= & \left(R_{j}^{-1} \hat{u}_{j, t}\right)^{2} \\
& -\left(\mathcal{A}^{-1}\left(\sum_{k=1}^{n} \Psi_{u_{k}} r_{k, 1}\left(R_{k}^{-1} \hat{u}_{k, t}\right)^{1}+\sum_{k=1}^{n} \Psi_{u_{k}} r_{k, 2}\left(R_{k}^{-1} \hat{u}_{k, t}\right)^{2}\right)\right)^{j} \\
= & \left(R_{j}^{-1} \hat{u}_{j, t}\right)^{2}-\left(\mathcal{A}^{-1} \sum_{k=1}^{n} \Psi_{u_{k}} r_{k, 1}\left(R_{k}^{-1} \hat{u}_{k, t}\right)^{1}\right)^{j} \\
& -\left(\mathcal{A}^{-1} \mathcal{A}\left(\begin{array}{c}
\left(R_{1}^{-1} \hat{u}_{1, t}\right)^{2} \\
\vdots \\
\left(R_{n}^{-1} \hat{u}_{n, t}\right)^{2}
\end{array}\right)\right)^{j} .
\end{aligned}
$$

Summarizing, we observe that the previous computation of the second component of $\frac{d}{d t} v_{j}\left(t^{m}\right)$ up to $O(\Delta x)^{2}$ with equation (20) and (22). Hence, the proposed method slightly improves the construction presented in [6]. Compared with [6] it also does not require during the construction information on $\left(R_{j}^{-1} \hat{u}_{j, t}\right)^{2}$ being the information on the outgoing characteristic on arc $j$.

\section{Application to gas DYNAMics}

We discuss the application of the method to gas dynamics in connected pipe systems. Those problems have been studied intensively in the past years and we refer e.g. to $[2,3,13,15-18]$ for analytical and computational results. Here, we study a single node with $n$ connected arcs and on each arc the dynamics are governed by the isentropic Euler equations (38) (or the $p$-system). We assume a subsonic state $\hat{u}=\left(\hat{u}_{j}\right)_{j=1}^{n}$ is given such that condition (4) is fulfilled. The wave curves and properties of the isentropic Euler equations are well known [20] and they are given on arc $j$ by

$$
0=\partial_{t} \rho_{j}+\partial_{x} q_{j} \text { and } 0=\partial_{t} q_{j}+\partial_{x}\left(p\left(\rho_{j}\right)+\frac{q_{j}^{2}}{\rho_{j}}\right) .
$$

Here, $p(\cdot) \in C^{2}$ is the pressure law which is supposed to be non-negative, strictly monotone increasing and convex, with e.g. $p(\rho)=a^{2} \rho$ being a model for isothermal gas. The subsonic region is the set $\Omega:=\left\{(\rho, q): \rho>0, \lambda^{1}(\rho, q)<0<\lambda^{2}(\rho, q)\right\}$ where 
$\lambda^{1,2}=\frac{q}{\rho} \mp \sqrt{p^{\prime}(\rho)}$. The corresponding (right) eigenvectors are

$$
r_{1}(\rho, q)=\left(\begin{array}{c}
-1 \\
-\lambda^{1}(\rho, q)
\end{array}\right) \text { and } r_{2}(\rho, q)=\left(\begin{array}{c}
1 \\
\lambda^{2}(\rho, q)
\end{array}\right)
$$

The reversed 2-Lax curve exiting at $(\hat{\rho}, \hat{q})$ is given by

$$
\mathcal{L}_{2}(\hat{u}, \sigma)=\left(\begin{array}{cc}
\sigma \frac{\hat{q}}{\hat{\rho}}+\sqrt{\frac{\sigma}{\hat{\rho}}(\sigma-\hat{\rho})(p(\sigma)-p(\hat{\rho}))}, & \sigma>\hat{\rho} \\
\sigma \frac{\hat{q}}{\hat{\rho}}-\sigma \int_{\sigma}^{\hat{\rho}} \frac{\sqrt{p^{\prime}(s)}}{s} d s, & \sigma \leq \hat{\rho}
\end{array}\right) .
$$

Different coupling conditions have been proposed in the literature [2]. Common to all is the conservation of mass flux across the node. Hence, the first component of $\Psi$ reads

$$
\Psi^{1}(\hat{u}(t, 0+))=\sum_{j=1}^{n} \hat{q}_{j}(t, 0+) .
$$

The remaining $n-1$ conditions can be prescribed for example by the equality of the pressure

$$
\Psi^{j}(\hat{u}(0+, t))=p\left(\hat{\rho}_{j}(0+, t)\right)-p\left(\hat{\rho}_{j-1}(0+, t)\right), j=2, \ldots, n .
$$

Remark 4.1. Let $n=2$. Consider coupling condition (40) and the equality of momentum

$$
\Psi^{2}(\hat{u}(0+, t))=p\left(\hat{\rho}_{2}(0+, t)\right)+\frac{\hat{q}_{2}^{2}(0+, t)}{\hat{\rho}_{2}(0+, t)}-p\left(\hat{\rho}_{1}(0+, t)\right)-\frac{\hat{q}_{1}^{2}(0+, t)}{\hat{\rho}_{1}(0+, t)} .
$$

We recover a solution $(\bar{\rho}, \bar{q})(x, t)$ to the Cauchy problem

$$
0=\partial_{t} \bar{\rho}+\partial_{x} \bar{q}, 0=\partial_{t} \bar{q}+\partial_{x}\left(p(\bar{\rho})+\frac{\bar{q}^{2}}{\bar{\rho}}\right), x \in \mathbb{R}, t \geq 0,
$$

by $\bar{q}(x, t)=-q_{1}(-x, t)$ and by $\bar{q}(x, t)=q_{2}(x, t)$ for $x \in \mathbb{R}_{0}^{+}$. For details we refer to [14].

For the reconstruction of $v_{j}(t)$ in STEP2, we collect the following elementary computations. Equation (15) is solved for $s \in \mathbb{R}^{n}$ using Newton's method with $\mathcal{L}_{2}(\hat{u}, s)$ given by equation (39) and $\Psi$ given by (40) and (41), respectively. This enables us to determine $v_{j}\left(t^{m}\right)$ according to (16). In order to obtain $\partial_{t} v_{j}\left(t^{m}\right)$, we solve equation (19) with $R_{j}=\left(r_{1}\left(v_{j}\left(t^{m}\right)\right), r_{2}\left(v_{j}\left(t^{m}\right)\right)\right)$. For the second component we note that

$$
\begin{aligned}
& \left(D_{u_{1}} \Psi(\hat{u}) r_{2}\left(\hat{u}_{1}\right), \ldots, D_{u_{n}} \Psi(\hat{u}) r_{2}\left(\hat{u}_{n}\right)\right)
\end{aligned}
$$

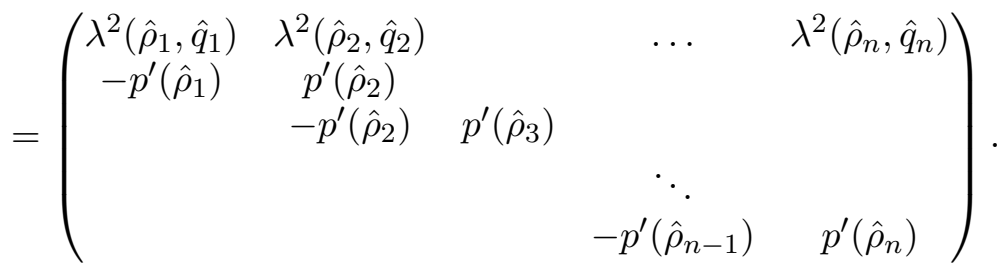


Further, for $\hat{u}:=\left(v_{j}\left(t^{m}\right)\right)_{j=1}^{n}$ we compute the vector

$$
\begin{aligned}
& \sum_{k=1}^{n} D_{k} \Psi(\hat{u}) r_{1}\left(\hat{u}_{k}\right) \lambda^{1}\left(\hat{u}_{k}\right)\left(R_{k}^{-1} \sigma_{k, 0}\right)^{1}
\end{aligned}
$$

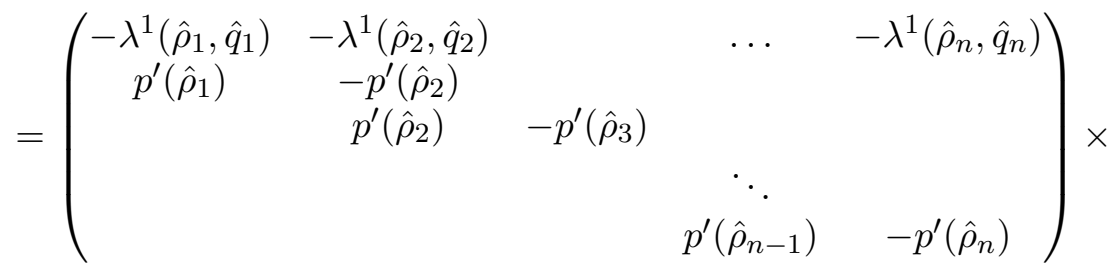

$$
\begin{aligned}
& \left(\begin{array}{c}
\lambda^{1}\left(\hat{u}_{1}\right)\left(R_{1}^{-1} \sigma_{1,0}\right)^{1} \\
\vdots \\
\lambda^{1}\left(\hat{u}_{n}\right)\left(R_{n}^{-1} \sigma_{n, 0}\right)^{1}
\end{array}\right) .
\end{aligned}
$$

The previous computations enable us to solve equation (20) for $\partial_{t} \mathbf{v}_{k}^{2}\left(t^{m}\right)$ and hence to determine $\frac{d}{d t} v_{j}\left(t^{m}\right)$ by equation (22).

\section{Computational Results}

\subsection{Linear and node vs linear on a single arc.}

Here we will show numerical results on a linear transport equation as (1) for a single arc (therefore, we drop the index $j$ ).

The setup is a periodic domain $x \in[0,2 \pi]$ (i.e. for the state $u$ we have $u(0, t)=$ $u(2 \pi, t), \forall t \geq 0)$, as flux we set $f \equiv 1$, and the initial condition (2) is picked as $u_{o}(x)=$ $\sin (x)$.

For the nodal solution on the domain $[0,2 \pi]$ we coupled the arc with itself and we used $\phi$ as in (25), i.e. $\Psi(u(t, 0+), u(t, 2 \pi-))=u(t, 0+)-u(t, 2 \pi-)=0 \forall t \geq 0$.

Furthermore, the Courant number is set to 0.9 and the following examples are grounded on a final time $T=10$. For both scenarios we implementet a first order accurate Upwind scheme as well as a MUSCL scheme [43], where we used the modified MUSCL scheme as in (31) in the coupled case. The following will illustrate both scheme in the spacial cyclic and in the nodal coupled case. The power $k$ of $N_{k}=2^{k}$ (the number of degrees of freedom of the spacial discretization) we plotted against $\frac{\log \left(e_{N_{k}}\right)}{\log (2)}$, where $e_{N_{k}}$ is the $L^{1}$ error to the analytical solution for a given $N_{k}$.

Here, Table 5.1 contains the $L^{1}$ errors and convergence rate of the upper plots. $N_{k}$ and $r_{k}=\frac{\log \left(e_{N_{k}}\right)}{\log (2)}$ as above. The "Rate" columns are therefore $r_{k}-r_{k+1}$ of each error.

The numerical results are in accordance with the theoretical prediction. We observe the second-order convergence of the MUSCL scheme with nodal coupling conditions as proven. In the case of two connected arcs the nodal problem is formally equivalent to a periodic problem. Numerically, we observe that the rates are almost equivalent to the implementation of a periodic MUSCL scheme. This indicates the validity of the presented approach. 


\begin{tabular}{|c|c|c|c|c|c|c|}
\hline$N_{k}$ & Upwind $r_{k}$ & Rate & MUSCL periodic $r_{k}$ & Rate & MUSCL nodal $r_{k}$ & Rate \\
\hline $2^{5}$ & -0.7428 & $/$ & -3.0768 & $/$ & -3.1472 & $/$ \\
$2^{6}$ & -1.5442 & 0.8014 & -4.6573 & 1.5805 & -4.7055 & 1.5583 \\
$2^{7}$ & -2.4380 & 0.8938 & -6.4464 & 1.7891 & -6.4689 & 1.7635 \\
$2^{8}$ & -3.3842 & 0.9462 & -8.3080 & 1.8616 & -8.3198 & 1.8509 \\
$2^{9}$ & -4.3577 & 0.9735 & -10.1955 & 1.8876 & -10.2018 & 1.8820 \\
$2^{10}$ & -5.3440 & 0.9863 & -12.1139 & 1.9184 & -12.1171 & 1.9153 \\
$2^{11}$ & -6.3372 & 0.9932 & -14.0533 & 1.9394 & -14.0549 & 1.9378 \\
$2^{12}$ & -7.3339 & 0.9967 & -16.0073 & 1.9540 & -16.0081 & 1.9532 \\
$2^{13}$ & -8.3322 & 0.9983 & -17.9715 & 1.9642 & -17.9719 & 1.9638 \\
$2^{14}$ & -9.3313 & 0.9991 & -19.9429 & 1.9714 & -19.9431 & 1.9712 \\
\hline
\end{tabular}

TABle 1. Comparison of convergence of the periodic boundary to the nodal coupled method

5.2. Gas dynamics. We compute the conservation law given by equation (38) and consider a comparison with a periodic case as well as a true network.

The spatial domain is $x \in[0,2 \pi]$ with $u(0, t)=u(2 \pi, t) \forall t \geq 0$. For comparison we compute a second order MUSCL scheme with periodic boundary conditions to obtain a trues̈olution. In order to compare the coupled case with the periodic case we consider two arcs $j=1$ and $j=2$. The domain of arc $j=1$ is $x \in[0, \pi]$ and the domain of arc $j=2$ is $[\pi, 2 \pi]$. They are coupled twice, once at $x=0$ and at $x=\pi$. For reasons of visual comparison we depict density and flux on the full domain $x \in[0,2 \pi]$. In order to have consistency with the periodic situation we impose the following coupling condition: As first component of the coupling $\Psi$ the conservation of mass (40) and as second component we use the conservation of momentum as suggested in Remark 4.1. The second condition leads to a true nonlinear coupling compared with the approach by [6].

As initial data we set

$$
U(x, 0)=\left(\begin{array}{l}
\rho(x, 0) \\
q(x, 0)
\end{array}\right)=\left(\begin{array}{c}
0.1 \cos (x)+1 \\
0.05 \cos (x)+2
\end{array}\right) .
$$

The final time is $T=0.4$. The chosen pressure law is

$$
p(\rho)=a^{2} \rho \text { with } a=5 .
$$

We compare the periodic standard second order solution $U_{c y c}=\left(\rho_{c y c}, q_{c y c}\right)^{\top}$ to the coupled solution $U_{c c}=\left(\rho_{c c}, q_{c c}\right)^{\top}$ in Figure 5.2. We observe no difference between both solutions as expected. Next, we investigate the convergence rate in $L^{1}$ and $L^{\infty}$-norm.

The Table 5.2 contains the $L^{1}$ errors and convergence rate of the coupled solution compared to the periodic case. $N_{k}=2^{k}$ is the number of degrees of freedom due to the spatial discretization. The column " $L^{1} \rho "$ gives $r_{\rho_{k}}=\log \left(\left\|\rho_{c y c}-\rho_{c c}\right\|_{L^{1}}\right) / \log (2)$ and $" L^{1} q " r_{q_{k}}=\log \left(\left\|q_{c y c}-q_{c c}\right\|_{L^{1}}\right) / \log (2)$ analogously. The "Rate" columns are therefore $r_{k}-r_{k+1}$ of each error of both quantities $\rho$ and $q$. 

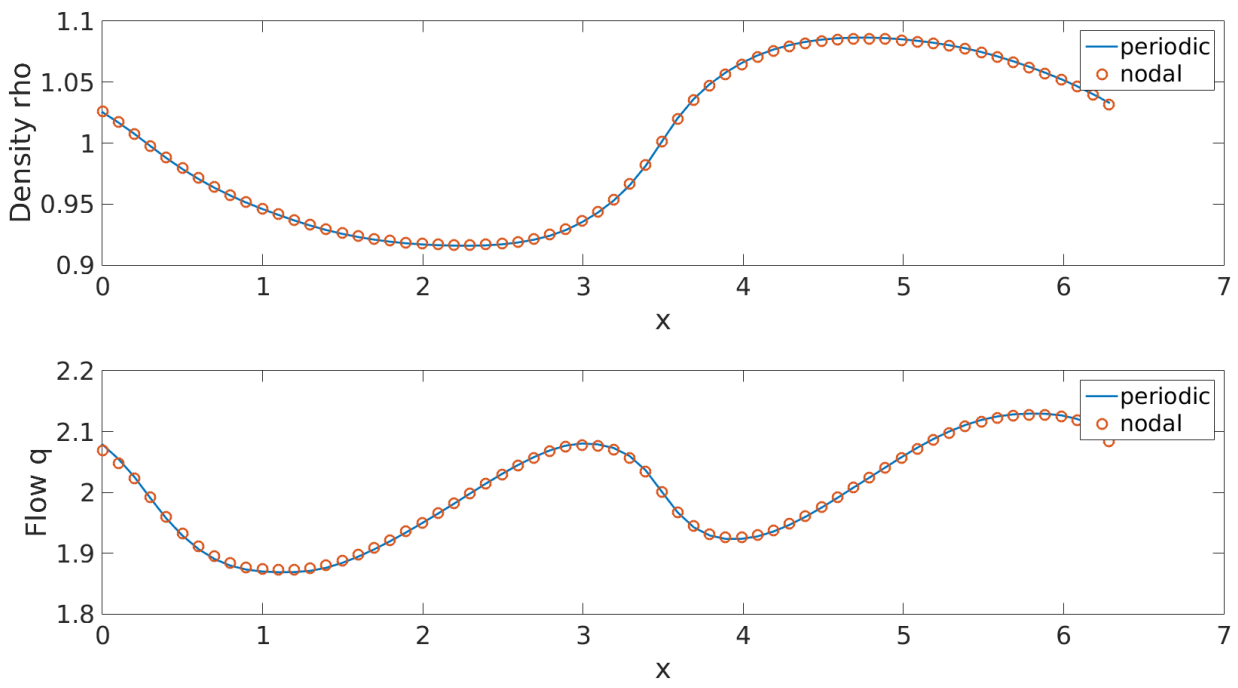

Figure 2. Periodic versus nodal coupled solution

\begin{tabular}{|l||r|r||c|c|}
\hline$N_{k}$ & $L^{1} \rho$ & $L^{1} q$ & Rate $\rho$ & Rate $q$ \\
\hline $2^{5}$ & -8.8259 & -7.1826 & $/$ & $/$ \\
$2^{6}$ & -10.4556 & -8.6067 & 1.6297 & 1.4242 \\
$2^{7}$ & -12.1436 & -10.3566 & 1.6880 & 1.7499 \\
$2^{8}$ & -13.8907 & -12.1301 & 1.7471 & 1.7735 \\
$2^{9}$ & -15.6996 & -13.9506 & 1.8089 & 1.8205 \\
$2^{10}$ & -17.5927 & -15.8745 & 1.8931 & 1.9239 \\
$2^{11}$ & -19.5538 & -17.8503 & 1.9611 & 1.9758 \\
$2^{12}$ & -21.5445 & -19.8373 & 1.9907 & 1.9870 \\
\hline
\end{tabular}

TABLE 2. $L^{1}$ convergence of the periodic boundary to the nodal coupled method

The Table 5.3 contains the $L^{\infty}$ errors and convergence rate of the coupled solution. The scenario is the same as in Table 5.2 but all data are presented under the $L^{\infty}$ norm. We observe here, that the scheme is first order only. This is due to the local first order accuracy of the TVD method at a local extremum [26]).

Finally, we compare the solution at time $T=1$ with a solution obtained through a first-order scheme in Figure 1. Here, we use the same implementation but all numerical slopes are equal to zero. As expected the first order scheme has a high diffusion leading to a diffusive behavior. This can in particular be seen in the evolution of the flux $q$ which is significantly reduced close to local extrema.

Summarizing, we observe that the presented method to obtain a coupled second-order scheme does yield the expected results. The solution does not differ from a periodic numerical solution, it reaches second-order convergence in $L^{1}$ and slightly smaller order in 
$L^{\infty}$. The relevance of the second-order is seen in Figure 1 where the numerical diffusion damps the local extrema in the flux $q$ significantly in the case of the first-order scheme.

\begin{tabular}{|l||r|r||c|c|}
\hline$N_{k}$ & $L^{\infty} q$ & $L^{\infty} \rho$ & Rate $\rho$ & Rate $q$ \\
\hline $2^{5}$ & -8.1963 & -5.1755 & $/$ & $/$ \\
$2^{6}$ & -9.2077 & -6.1543 & 1.0114 & 0.9788 \\
$2^{7}$ & -10.1478 & -7.1371 & 0.9402 & 0.9828 \\
$2^{8}$ & -10.9934 & -8.0115 & 0.8456 & 0.8745 \\
$2^{9}$ & -11.9733 & -9.0249 & 0.9799 & 1.0134 \\
$2^{10}$ & -13.0005 & -10.0815 & 1.0272 & 1.0566 \\
$2^{11}$ & -13.9772 & -11.0764 & 0.9767 & 0.9948 \\
$2^{12}$ & -14.9497 & -12.0575 & 0.9725 & 0.9811 \\
\hline
\end{tabular}

TABLE $3 . L^{\infty}$ convergence of the periodic boundary to the nodal coupled method
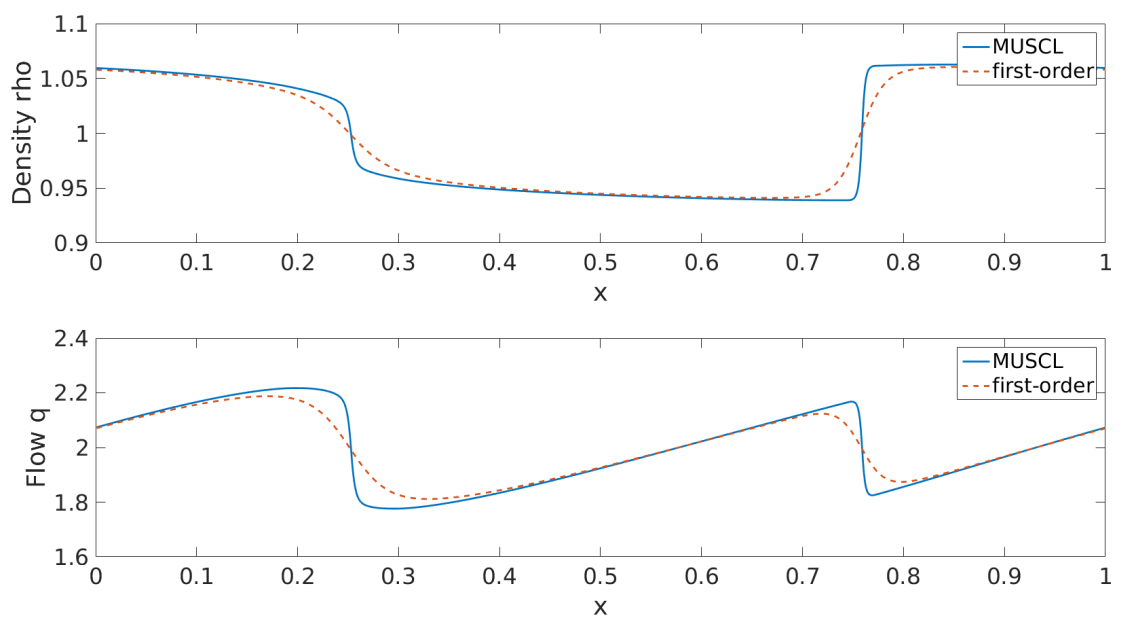

Figure 3. Nodal case at $T=1$ and for $N=1024$ gridpoints computed with a first-order (dashed) and second-order MUSCL scheme (line). In the left figure we show the density $\rho$ and in the right figure the flux $q$.

5.3. Gas dynamics - Y-junction. Here we have a look at the setup as suggested in Section 2 for $n=3$ (number of arcs attached to the node at $x=0$ ). We consider a spatial domain, $x \in[0,2]$ for all arcs. For the boundary which is not adjacent to the coupled knot we implemented Neumann boundary conditions. The chosen coupling conditions are the conservation of mass (40) and conservation of momentum 4.1 as in the previous example.

The initial data are as follows:

For the first arc $j=1$ we set

$$
U_{1}(x, 0)=\left(\begin{array}{c}
\rho_{1}(x, 0) \\
q_{1}(x, 0)
\end{array}\right)
$$


with

$$
\rho_{1}(x, 0)= \begin{cases}-x^{3}+\frac{3}{2} x^{2}+1 & x \in[0,1) \\ \frac{3}{2} & x \in[1,2]\end{cases}
$$

and

$$
q_{1}(x, 0)=\left\{\begin{array}{ll}
-x^{3}+\frac{3}{2}(x-1)^{2} & x \in[0,1) \\
\frac{1}{2} & x \in[1,2]
\end{array} .\right.
$$

For $j=2,3$ we have

$$
U_{j}(x, 0)=\left(\begin{array}{c}
\rho_{j}(x, 0) \\
q_{j}(x, 0)
\end{array}\right),
$$

with

$$
q_{j}(x, 0) \equiv 0
$$

and

$$
\rho_{j}(x, 0) \equiv 1 .
$$

Those initial data are smooth and give us a feasible initial state with respect to the coupling condition. Hence, $\Psi\left(U_{1}(x, 0), U_{2}(x, 0), U_{3}(x, 0)\right)=(0,0,0)^{\top}$.

The chosen pressure law is again

$$
p(\rho)=a^{2} \rho \text { and } a=5
$$

and the final time is set to $T=0.3$. The remaining numerics are the same as in Section 5.2 .
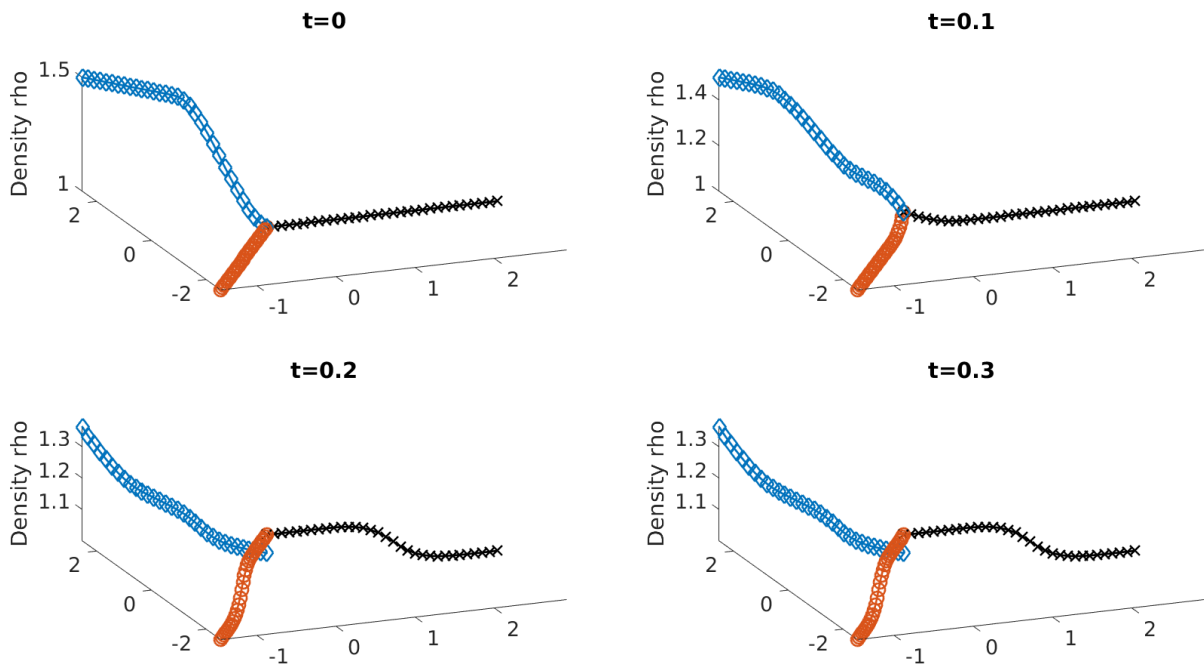

Figure 4. Evolution of the density $\rho$. Arcs: $\diamond j=1, \circ j=2, \times j=3$. 

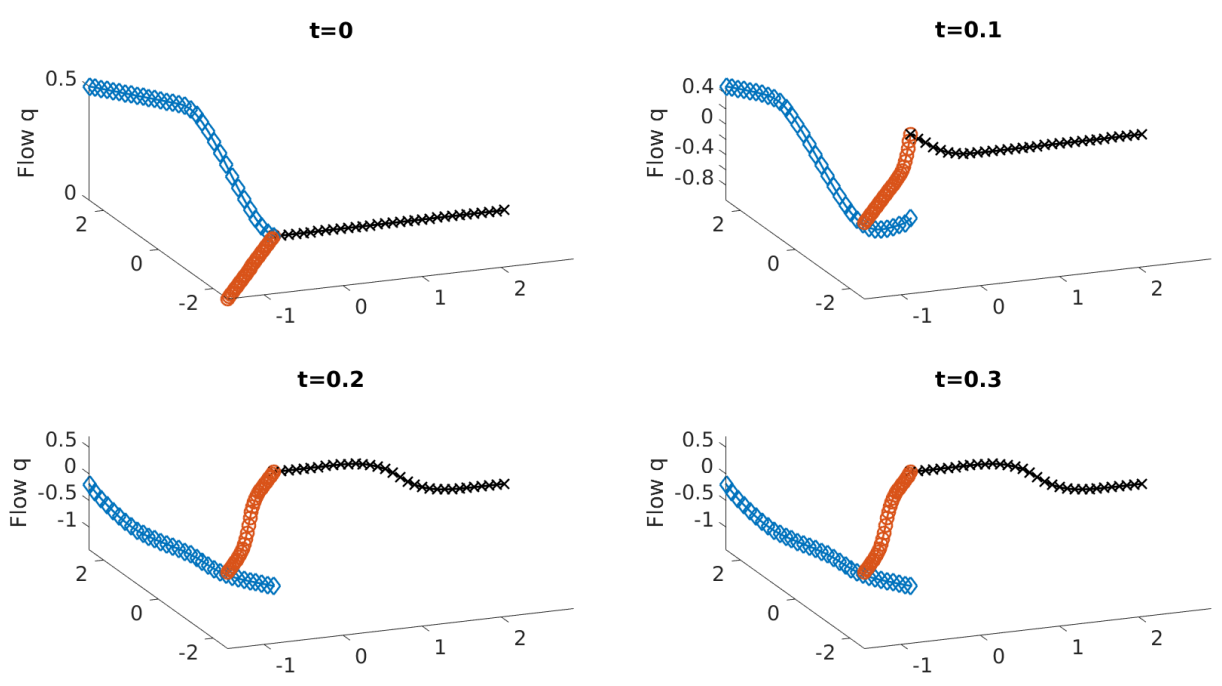

Figure 5. Evolution of the flow $q$. Arcs: $\diamond j=1, \circ j=2, \times j=3$.

The Table 5.4 contains the $L^{1}$ errors and convergence rate of the coupled solution compared to a numerical solution of higher discretization at each arc $(j=1,2,3)$. The errors and order are computed analogously to table 5.2. Since the initial data in arc $j=2$ and $j=3$ are identical we get coinciding data for those both arcs. Hence, we only show one data set in the convergence table below.

We observe that also in the case of a network we obtain the second-order convergence rate with the suggested implementation of the scheme. From Figure 5.3 we observe that the total mass is conserved since the sum of the fluxes at $x=0$ equals zero. Also, the propagation of waves out of the node is visible; in particular, when considering at $j=3$. Initially, there was constant data given and slowly a wave emerges due to the interaction at the nodal point. This wave moves out of the node and reaches the midpoint of the third arc at time $t=0.3$. In the other arcs a similar behavior is present. 


$\mid$\begin{tabular}{|l||r|r||c|c|}
\hline$j=1: N_{k}$ & $L^{1} \rho$ & $L^{1} q$ & Rate $\rho$ & Rate $q$ \\
\hline $2^{5}$ & -7.6683 & -5.6102 & $/$ & $/$ \\
$2^{6}$ & -9.2223 & -7.1885 & 1.5541 & 1.5783 \\
$2^{7}$ & -10.7893 & -8.7746 & 1.5669 & 1.5861 \\
$2^{8}$ & -12.3244 & -10.3241 & 1.5351 & 1.5494 \\
$2^{9}$ & -13.7622 & -11.7769 & 1.4378 & 1.4529 \\
$2^{10}$ & -15.1328 & -13.1563 & 1.3706 & 1.3794 \\
$2^{11}$ & -16.5236 & -14.5467 & 1.3908 & 1.3904 \\
$2^{12}$ & -18.1942 & -16.2137 & 1.6706 & 1.6671 \\
\hline$j=2,3: N_{k}$ & $L^{1} \rho$ & $L^{1} q$ & Rate $\rho$ & Rate $q$ \\
\hline $2^{5}$ & -8.8909 & -6.5146 & $/$ & $/$ \\
$2^{6}$ & -10.5499 & -8.1738 & 1.6591 & 1.6593 \\
$2^{7}$ & -12.4446 & -10.0709 & 1.8947 & 1.8970 \\
$2^{8}$ & -14.3670 & -11.9932 & 1.9223 & 1.9223 \\
$2^{9}$ & -16.3003 & -13.9261 & 1.9333 & 1.9329 \\
$2^{10}$ & -18.2503 & -15.8749 & 1.9500 & 1.9488 \\
$2^{11}$ & -20.2580 & -17.8813 & 2.0077 & 2.0064 \\
$2^{12}$ & -22.4155 & -20.0355 & 2.1575 & 2.1542 \\
\hline
\end{tabular}

TABLE 4. $L^{1}$ convergence on a network with $j=1, \ldots, 3$ arcs

Further examples of coupled dynamics would be shallow-water equations in open canals. Those are similar to the case of gas dynamics using as " pressure law" $p(\rho)=\rho^{2}$. Due to the generality of the presented approach we do not expect any difference in the results compared with the shown examples. Among other examples for $2 \times 2$ conservation laws are traffic flow equations like the Aw-Rascle-Zhang model [1]. Coupling conditions for this model can be found e.g. in [24,31].

\section{Conclusion}

We presented a numerical method to implement coupling conditions in high-order finite volume schemes. The applied procedure is similar to the approach presented in [39], see Remark 2.4. However, therein, a fixed boundary condition is considered, while here an additional nonlinear dependence between states on different arcs is imposed. This leads to implicit boundary conditions. The discussion treats the case of a single node connected to $n$ arcs with nonlinear condition imposed at the node. The presented method is proved to be consistent in the case $n=2$ with a high-order discretization of a Cauchy problem. The presented method shows the expected convergence behavior in the linear case as well as in the fully nonlinear case of gas dynamics with nonlinear coupling for multiple attached arcs. So far, only results for second-order and $2 \times 2$ hyperbolic conservation have been addressed. However, the general method is extendable to $k \times k$ conservation laws as well as higher order methods that will be discussed in future work.

Acknowledgments. This work has been partly supported by DFG 'Integrated Production in High-Wage Countries' and the BMBF KinOpt project. This work is also based on the research supported in part by the National Research Foundation of South Africa 
(Grant Number: 93476) and the Research Development Programme of the University of Pretoria.

\section{REFERENCES}

[1] A. Aw And M. RASCle, Resurection of second order models of traffic flow, SIAM J. Appl. Math., 60 (2000), pp. 916-944.

[2] M. K. BAnda, M. Herty, And A. Klar, Coupling conditions for gas networks governed by the isothermal Euler equations, Netw. Heterog. Media, 1 (2006), pp. 295-314 (electronic).

[3] —_, Gas flow in pipeline networks, Netw. Heterog. Media, 1 (2006), pp. 41-56.

[4] G. Bastin, J. Coron, And B. DAndréa Novel, Using hyperbolic systems of balance laws for modeling, control and stability analysis of physical networks, in Lecture notes for the Pre-congress workshop on complex embedded and networked control systems, 17th IFAC World Congress, 2008.

[5] G. Bastin, J. Coron, AND B. DAndrÉa Novel, On lyapunov stability of linearised saint-venant equations for a sloping channel, Networks Heterogeneous Media, 4 (2009), pp. 177-187.

[6] R. Borsche And J. Kall, Ader schemes and high order coupling on networks of hyperbolic conservation laws, Journal of Computational Physics, 273 (2014), pp. 658-670.

[7] A. Bressan, S. Canic, M. Garavello, M. Herty, and B. Piccoli, Flow on networks: recent results and perspectives, European Mathematical Society-Surveys in Mathematical Sciences, 1 (2014), pp. $47-11$.

[8] G. Bretti, R. Natalini, And B. Piccoli, Fast algorithms for the approximation of a traffic flow model on networks., Discrete Contin. Dyn. Syst., Ser. B, 6 (2006), pp. 427-448.

[9] - Fast algorithms for the approximation of a traffic flow model on networks, Discrete Contin. Dyn. Syst. Ser. B, 6 (2006), pp. 427-448 (electronic).

[10] G. Bretti, R. Natalini, And B. Piccoli, Numerical approximations of a traffic flow model on networks, Netw. Heterog. Media, 1 (2006), pp. 57-84.

[11] J. Brouwer, I. Gasser, And M. Herty, Gas pipeline models revisited: model hierarchies, nonisothermal models, and simulations of networks, Multiscale Model. Simul., 9 (2011), pp. 601-623.

[12] R. Colombo And M. Garavello, A well posed riemann problem for the p-system at a junction, Netw. Heterog. Media, 1 (2006), pp. 495-511.

[13] R. M. Colombo and M. Garavello, On the p-system at a junction, in Control methods in PDE-dynamical systems, vol. 426 of Contemp. Math., Amer. Math. Soc., Providence, RI, 2007, pp. 193-217.

[14] — On the Cauchy problem for the p-system at a junction, SIAM J. Math. Anal., 39 (2008), pp. $1456-1471$.

[15] R. M. Colombo, G. Guerra, M. Herty, and V. Schleper, Optimal control in networks of pipes and canals, SIAM J. Control Optim., 48 (2009), pp. 2032-2050.

[16] R. M. Colombo, M. Herty, And V. SAchers, On $2 \times 2$ conservation laws at a junction, SIAM J. Math. Anal., 40 (2008), pp. 605-622.

[17] R. M. Colombo and F. Marcellini, Coupling conditions for the $3 \times 3$ Euler system, Netw. Heterog. Media, 5 (2010), pp. 675-690.

[18] R. M. Colombo And C. Mauri, Euler system at a junction, Journal of Hyperbolic Differential Equations, 5 (2007), pp. 547-568.

[19] R. Courant, K. O. Friedrichs, and H. Lewy, Über die partiellen differenzengleichungen der mathematischen physik, Mathematische Annalen, 100 (1928), pp. 32-74.

[20] C. M. Dafermos, Hyperbolic conservation laws in continuum physics, vol. 325 of Grundlehren der Mathematischen Wissenschaften [Fundamental Principles of Mathematical Sciences], SpringerVerlag, Berlin, second ed., 2005.

[21] C. D'Apice, S. Göttlich, M. Herty, and B. Piccoli, Modeling, simulation, and optimization of supply chains, Society for Industrial and Applied Mathematics (SIAM), Philadelphia, PA, 2010. A continuous approach.

[22] C. D'Apice, R. Manzo, And B. Piccoli, A fluid dynamic model for telecommunication networks with sources and destinations, SIAM J. Appl. Math., 68 (2008), pp. 981-1003. 
[23] C. D'Apice, R. Manzo, And B. Piccoli, Existence of solutions to Cauchy problems for a mixed continuum-discrete model for supply chains and networks, J. Math. Anal. Appl., 362 (2010), pp. 374386.

[24] M. Garavello And B. Piccoli, Traffic flow on a road network using the Aw-Rascle model, Comm. Partial Differential Equations, 31 (2006), pp. 243-275.

[25] — Traffic flow on networks, vol. 1 of AIMS Series on Applied Mathematics, American Institute of Mathematical Sciences (AIMS), Springfield, MO, 2006. Conservation laws models.

[26] E. Godlewski And P.-A. Raviart, Hyperbolic Systems of Conservation Laws, Mathematiques and Applications, Ellipses, first ed., 1991.

[27] S. K. Godunov, A difference scheme for the numerical computation of a discontinuous solution of the hydrodynamic equation, Math. Sbornik, 47 (1959), pp. 271-306.

[28] M. Gugat, Optimal nodal control of networked hyperbolic systems: evaluation of derivatives, Adv. Model. Optim., 7 (2005), pp. 9-37 (electronic).

[29] B. Haut And G. Bastin, A second order model of road junctions in fluid models of traffic networks., Netw. Heterog. Media, 2 (2007), pp. 227-253.

[30] M. Herty, C. Jörres, AND B. PicCOLI, Existence of solution to supply chain models based on partial differential equation with discontinuous flux function, J. Math. Anal. Appl., 401 (2013), pp. 510-517.

[31] M. HeRty AND M. RASCle, Coupling conditions for a class of second-order models for traffic flow, SIAM J. Math. Anal., 38 (2006), pp. 595-616.

[32] S. Jin AND Z. P. XIN, The relaxation schemes for systems of conservation laws in arbitrary space dimensions, Comm. Pure Appl. Math., 48 (1995), pp. 235-276.

[33] O. Kolb, J. LANG, AND P. BAlEs, An implicit box scheme for subsonic compressible flow with dissipative source term, Numer. Algorithms, 53 (2010), pp. 293-307.

[34] D. KRÖNER, Numerical schemes for conservation laws, Wiley Teubner, 1997.

[35] G. Leugering And J. Schmidt, On the modelling and stabilization of flows in networks of open canals, SIAM journal on control and optimization, 41 (2002), p. 164.

[36] R. J. LeVEque, Finite volume methods for hyperbolic problems, Cambridge Texts in Applied Mathematics, Cambridge University Press, 2002.

[37] H. Nessyahu And E. TADmor, Nonoscillatory central differencing for hyperbolic conservation laws, J. Comput. Phys., 87 (1990), pp. 408-463.

[38] P. SweBY, High resolution schemes using flux limiters for hyperbolic conservation laws, SIAM Journal on Numerical Analysis, (1984), pp. 995-1011.

[39] S. TAN AND C.-W. SHU, Inverse Lax-Wendroff procedure for numerical boundary conditions of conservation laws, J. Comput. Phys., 229 (2010), pp. 8144-8166.

[40] - Inverse Lax-Wendroff procedure for numerical boundary conditions of hyperbolic equations: survey and new developments, in Advances in applied mathematics, modeling, and computational science, vol. 66 of Fields Inst. Commun., Springer, New York, 2013, pp. 41-63.

[41] S. TAN, C. Wang, C.-W. Shu, And J. Ning, Efficient implementation of high order inverse LaxWendroff boundary treatment for conservation laws, J. Comput. Phys., 231 (2012), pp. 2510-2527.

[42] E. F. Toro, Riemann Solvers and Numerical Methods for Fluid Dynamics: A Practical Introduction, Springer, second ed., 2009

[43] B. VAN LEER, Towards the ultimate conservative difference scheme. $v$. a second-order sequel to godunov's method, J. Comput. Phys., 32 (1979), pp. 101-136. 